\title{
A Multi-scale Morphological Algorithm for AFM Micrograph Edge Detection
}

\author{
Xue Gang FU \& Hua JIANG \\ Sichuan University of Science \& Engineering Dept. of Electronic Engineering, Zigong, Sichuan, China
}

\begin{abstract}
In this paper we present a new morphological edge detection method using a multi-scale approach. Edges of different fineness are detected using operator at different scale, and then they are regulated in order to eliminate the difference of gray attribution among multi-scale edges, and then combined to produce all the edges of interest in an image using the adaptive fusion method of minimums. The experiment results show that the fuzziness of image edge detection is reduced, and noise is removed efficiently, and distinct edges can be detected efficiently for micrograph.
\end{abstract}

KEYWORD: Micrograph; Edge detection; Multi-scale; Morphology

\section{GENERAL INSTRUCTIONS}

The invention of scanning tunneling microscope (STM) in 1982 and the world's first atomic force Microscope (AFM) in 1986 have been paid attention in many fields of science. The atomic force microscope is an image analysis instrument, which scan on the sample surface using the front sharp scanning probe of cantilever under the laser irradiation, and the light refraction in the contact area between samples and pinpoint is received through the photoelectric diode and then converted into recognizable information in image presentation. Microscopic objects after imaging using microscope is collected by CCD image sensor, then become micrograph. The micrographs are different from common images. The micrograph has a low contrast, tends to be fuzzy, and is easily affected by the light of microscope. The target object is not easy to identify from the background. Compared with the common pictures, the micrograph has the following characteristics: the scene structure is simple; Contrast is low; Been Vulnerable to be effected by the outside world lighting; Influence of the system's optical distortion is obvious; Noise has been amplified. Because of the restriction of microscope depth of focus, it does not well image in the outside part of the focus, and the fuzzy, deformation and a series of problems are presented. Due to the uneven illumination, the picture is easy to have noise and shadow in the process of microscopic imaging. The influence will be intensified after image amplification. Considering these characteristics, in the processing of micrograph, it is difficult to get satisfactory results with the common image edge detection algorithm [1-5]. It needs to choose the appropriate method according to the characteristics of microscopic image.

In this paper, we present a multi-scale morphological edge detection algorithm. Firstly, we use morphological edge detection operator to extract the image edge, then we fuse the image edge obtained with different scale through adaptive methods which can enhance the fuzzy edge and appropriately eliminate noise. Eventually, the experiments prove that the image edges are clear, and the noise elimination is obvious. Compared with other edge detection methods at the end of the article, the experiment results show that this method is feasible and effective.

\section{MATHEMATICAL MORPHOLOGY EDGE DETECTION ALGORITHM}

\subsection{Mathematical morphology edge detection algorithm}

The objects and characteristics in the image depend directly on the shape. The purpose of mathematical morphology is to research the shape in the time domain, so the morphology is suitable for image processing. The corrosion, expansion, opening and closing of morphological operation is the operation based on the operations of the set. The structural elements in these operations has a key role, which adjusts the geometric structure of image features' transformation. The classical differential operator detects [6-10] generally the discontinuity of local 
characteristics of image, and then puts these discontinuous edge pixels into complete border. But ,the noise has the characteristics that the gray scale changes quickly, so using differential operator to detect edge has the defects of promoting noise .If reduce noise, the target information is also removed. Mathematical morphology can be targeted to select structural element and the way of transformation, and also weak and strengthen a specific goal.

Edge detection operator based on gray scale morphological transformation has expansion edge detection operator, corrosion edge detection operator and morphology gradient edge detection operator. $\mathrm{f}$ represents gray scale image, g represents structural elements. $\oplus$ and $\odot$ represent respectively the operation of expansion and corrosion in morphological operation. (r,c) represents the coordinates of image pixels. These edge detection operators are respectively defined as follows:

Gray scale expansion edge detection operator

$$
C_{g d}=(f \oplus g)(r, c)-f(r, c)
$$

Gray scale corrosion edge detection operator

$$
C_{g e}=f(r, c)-(f \odot g)(r, c)
$$

Gray scale morphology gradient edge detection operator

$$
C_{\text {gsum }}=(f \oplus g)(r, c)-(f \odot g)(r, c)=C_{g d}+C_{g e}
$$

Equation(1) is based on morphological dilation. The signal of image edge is weak and there is the fuzzy edge of the image. Equation (2) is based on the morphological corrosion. Although the result of image edge signal is stronger, enhance the noise accordingly.

\subsection{Multi-scale morphological edge detection algorithm}

Structural elements is the key in morphological edge detection algorithm, it affects the image features and the direct result of image edge detection. The selection of different structural elements and different the size of the structure element can lead to different image operation results. We can make use of the different size of structural elements to extract image edge character. Although the ability that the structural elements of small size eliminate noises is weak, it can detect a better edge details. While the ability that the structural elements of large size eliminate noises is strong, it detects coarser edges. Combined edge image under the various different sizes can obtained multi-scale morphological edge detection algorithm.

The sequence $\left\{g_{i} \mid i=1,2, \cdots\right\}$ for a given structure element, if all have the same shape and the size increases monotonously with $\mathrm{i}$ is called for a multi- scale sequences. Sequence for a given structure element, different size of structural elements can be used to extract characteristics at different scales. In order to get the accurate information of edge detection and effectively overcome the impact of noise, we must be reasonable to adjust the size of the structural element.

When use multi-scale edge detection operator to detect image edge, the edge gray scale distribution obtained through the expansion or corrosion of the different scale will be different. .If we synthesize directly the edge of different scales, as well as do not consider the change of the gray scale distribution, it will make the edge fuzzy and not clear, and lost a lot of information.

\section{THE MULTI-SCALE MORPHOLOGICAL EDGE DETECTION ALGORITHM BASED ON FUSION}

When use multi-scale edge detection operator detect image edge, the edge gray scale distribution obtained through the different scale will be different, but the changing rule of the histogram is very similar. Based on this, we put forward a multi-scale edge detection algorithm based on the edge information fusion of different scale in this paper. Firstly, we extract the edge image with multi-scale structural elements morphological edge detection operator and normalize those edge images. Then ,fuse the image edge detected by the each size of structural element in the method of adaptive minimum to achieve the purpose of effectively enhance fuzzy edges and eliminate noise effects. The specific steps are as follows:

Step1: Detect respectively the image edge information with different scale structural elements.

1) Select a finite number of representative structural element.

In order to reduce that the detection operator is sensitive to noise, we detect the image edge in the way of expansion and corrosion in this article. There are six kinds of structural elements. They are respectively the prismatic structural elements,

$$
\text { Drod } 1=\{(0,-1),(0,1),(0,0),(-1,0),(1,0)\}
$$

The others,

$$
\begin{aligned}
& D 1=\{(-1,0),(0,0),(0,1)\} \quad D 2=\{(0,-1),(0,0),(1,0)\} \\
& D 3=\{(-1,0),(0,0),(0,-1)\} \quad D 4=\{(0,1),(0,0),(1,0)\} \\
& D 5=\{(-1,-1),(-1,1),(0,0),(1,-1),(1,1)\}
\end{aligned}
$$

Among them, the $(0,0)$ is the center of the structural elements.

2) The scale of multi-scale structural elements after various structural elements expanding is according to the specific circumstances.

3) We make a multi-scale edge extraction to the various structural elements of different scales .The 
expansion edge detection operator of the structural elements is as follows:

$$
G_{n d}^{\prime \prime}(x, y)=\min \left\{U_{n D r o d l}(x, y)-f(x, y), U_{n D 5}(x, y)-f(x, y), G_{n d}^{\prime}(x, y)\right\}
$$

\section{And}

$$
G_{n d}^{\prime}(x, y)=\max \left\{U_{n D 1}(x, y)-U_{n D 2}(x, y)|,| U_{n D 3}(x, y)-U_{n D 4}(x, y) \mid\right\}
$$

Step 2: We fuse the image edges detected adaptively by the each structural element of different scales. Then we obtain the synthetic edge image.

Step 2: We fuse the image edges detected adaptively by the each structural element of different scales.Then we obtain the synthetic edge image.

1) image normalization

When use multi-scale edge detection operator detect image edge, the edge gray scale distribution obtained through the different scale will be different, but the changing rule of the histogram is very similar. The purpose of image normalization is making the average gray level and contrast of the edge image of different scale be in a fixed level. $A(x, y)$ is on behalf of the gray value of original image on point $(x, y), B(x, y)$ is on behalf of the gray value of normalized image on point ${ }^{(x, y)}, \mathrm{M}$ and VAR represent respectively the mean and variance of the original fingerprint image, M0 and VAR0 represent respectively the mean and variance of the expectation. The pixel value of fingerprint image on point $(\mathrm{x}, \mathrm{y})$ after normalization is determined by the type:

$$
B(x, y)= \begin{cases}M 0+\sqrt{\frac{V A R 0(A(x, y)-M)^{2}}{V A R}} & A(x, y)>M \\ M 0-\sqrt{\frac{V A R 0(A(x, y)-M)^{2}}{V A R}} & A(x, y) \leq M\end{cases}
$$

2). The synthetic edge image

According to the following standards, we fuse various edge images.

$f^{\prime}(x, y)$ represents the new synthetic edge, $[\mathrm{K}, \mathrm{L}]$ represents the value rang, then

$$
f^{\prime}(x, y)=\min _{n=K}^{L} G_{n d}^{\prime \prime}(x, y)
$$

\section{RESULTS AND DISCUSSION}

In the experiment, we use the dropper to extract wine liquor and drop on the surface of the new mica plate. After drying in vacuum environment for 10 minutes, we catch better the microscopic structure of several types of liquor with the atomic force microtechnic of tapping mode of the SPA400 scanning probe microscope .We observe and compare the microscopic morphology of the different kinds and different flavor of wine, such as Luzhou laojiao, Guizhou maotai, wuliangye, 1573, Tang dynasty, Dong Jiu, Xushui daqu and so on. The results showed that all the wine have the distribution of the rules on the surface of mica plate, but the distributional pattern is very different, some are flower shape, some are dendritic, some are combing shape. The forms and structure are different and the structure are very clear.

Figure 1 (a) and figure 2 (a) are respectively microstructure of maotai and xushui daqu with 500 $\mathrm{nm} \times 500 \mathrm{~nm}$. As is shown in the picture, the image have the noise and shadow, and the image is fuzzy and not clear because of the influence of uneven illumination and background. We can use the algorithm proposed in this article to extract these microscopic image edge character, so that we can process it in late. In the simulation experiment, the scale of multi-scale expansion edge detection algorithm is 2.The selection of expected mean and variance should be the mean and variance of the original microscopic image in the image normalization processing of the edge fusion algorithm. Figure 1 and figure 2 is the result of the experiment. Figure $1(b, c)$ and figure $2(b, c)$ are respectively the result of edge detection on scale 1 , 2.we can see that the edge is vague, and the interference caused by illumination and background is obvious, the noise and shadow is also enlarged. Figure 1(d) and figure 2 (d) are respectively the fusion result of figure $1(\mathrm{~b}, \mathrm{c})$ and figure $2(\mathrm{~b}, \mathrm{c})$, and edge become clearer, the interference of illumination and background is reduced greatly. Figure 1(e) and figure 2 (e) are respectively the microscopic edge image of maotai and xushui daqu detected by Canny operator. Because of the low contrast of microscopic image and the influence of illumination and background, the edge detected by Canny operator is chaotic, and can not reflect the structure of microscopic image.

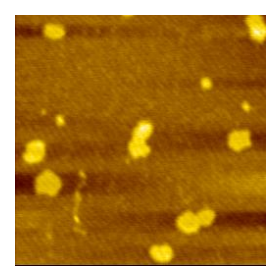

(a)

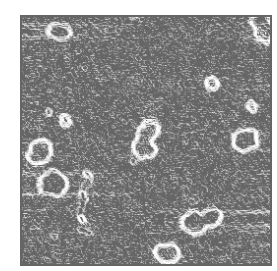

(b)

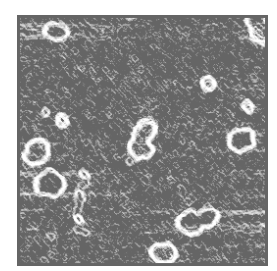

(c)

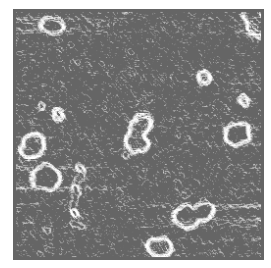

(d)

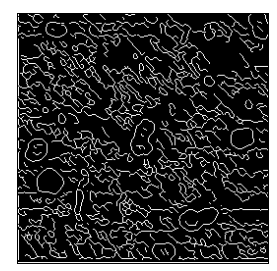

(e)
Figure 1 Maotai microscopic image edge extraction 


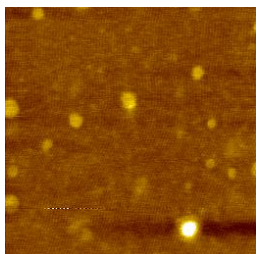

(a)

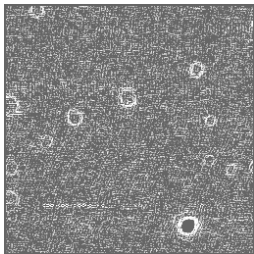

(b)

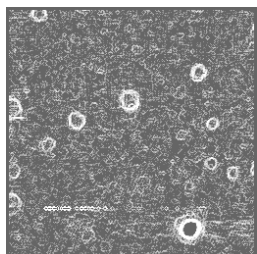

(c)

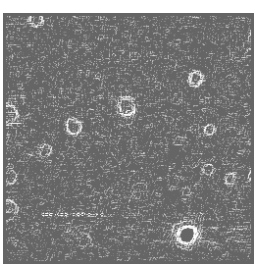

(d) (e)

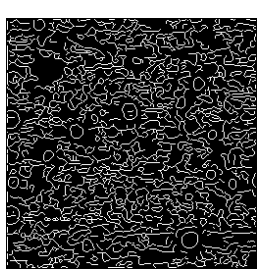

Figure2 Xu shui daqu microscopic image edge extraction

\section{CONCLUSIONS}

The microscopic image has a low contrast, tends to be fuzzy, and is easily affected by the light of microscope. The target object is not easy to identify from the background. Due to the uneven illumination, the picture is easy to have noise and shadow in the process of microscopic imaging. Considering these characteristics, in the processing of microscopic image, it is difficult to get satisfactory effect directly using common image edge detection algorithm. It needs to choose the appropriate method according to the characteristics of microscopic image. In this paper, we present a multi-scale morphological edge detection algorithm based on fusion, which detects respectively the image edge information of different scale of the microscopic image with different scale structural elements, then normalize those edge images, fuse adaptively the image edge detected by the each size of structural element in the way of minimum, obtain the ideal image edge, at the same time, enhance the fuzzy edges, and effectively eliminate the noise. The method proposed is very effective for microscopic image and the edge detected is very clear.

\section{ACKNOWLEDGMENT}

This work was supported by the Scientific Research Fund of Sichuan Provincial Artificial Intelligence Key Laboratory (2010RZ003, 2010RY003, 2011RYY02, 2011RYY04, 2012RYY05)

\section{REFERENCES}

[1] CUI Qi. Image Processing and Analysis ----Methods and Applications of Mathematic Morphologic. Beijing: Science Press, 2000.

[2] Mallat S, Hwang W L. Singularty Detection and Processing with Wavelets. IEEE Trans On Information Theory, 1992, 38(2): 617.

[3] HU Shou-ren. Application of ANN. Beijing: National Science and Technology Press, 1993.

[4] CHEN Wunfan. A New Algorithm of Edge Detection for Color Image: Generalized Fuzzy Operator. Science in China (A), 1995: 38(10):1272.

[5] CAO Han-qiang, ZHU Guang-xi. Image Edge Detection Based on IFS. Signal Processing, 1997, 13(4): 330.

[6] Bhabatosh Chanda,Malay K Kundu,Y Vani Padmaja. A Multi-scale Morphologic Edge Detection. Pattern Recognition, 1998, 31(10): 1469.

[7] LU Guan-ming, LI Shu-hong. Multi-scale Morphological Gradient Algorithm and It's Application in Image Segmentation. Signal Processing, 2001, 2(7): 37-41.

[8] J S J Lee. Morphologic edge detection. IEEE Trans on Robotics Automat, 1987, 3: 140 156.

[9] LIU Xun, YOU Zhi-sheng, An Image Edge Detection Based on Multi-scale Morphology. Opto-Electronic Engineering, 2003, 30(3): 56-58.

[10] LIU Zhi-fang, YOU Zhi-sheng, CAO Gang. A Multi-scale Color Vector Morphologic Edge Detection. Journal of Image and Graphics, 2002, 9(7): 888-893. 